\title{
Quantitative Nodule Detection in Low Dose Chest CT Scans: New Template Modeling and Evaluation for CAD System Design
}

\author{
Aly A. Farag ${ }^{1}$, Ayman El-Baz ${ }^{1}$, Georgy Gimel'farb ${ }^{2}$, \\ Mohamed Abou El-Ghar ${ }^{3}$, and Tarek Eldiasty ${ }^{3}$ \\ 1 CVIP Lab., University of Louisville, Louisville, KY 40292 \\ \{farag, elbaz\}@cvip.louisville.edu, http://www.cvip.louisville.edu \\ 2 Department of Computer Science, Tamaki Campus, \\ University of Auckland, Auckland, New Zealand \\ g.gimelfarb@auckland.ac.nz \\ 3 Mansoura University, Urology and Nephrology Center, Mansoura, Egypt
}

\begin{abstract}
Automatic diagnosis of lung nodules for early detection of lung cancer is the goal of a number of screening studies worldwide. With the improvements in resolution and scanning time of low dose chest CT scanners, nodule detection and identification is continuously improving. In this paper we describe the latest improvements introduced by our group in automatic detection of lung nodules. We introduce a new template for nodule detection using level sets which describes various physical nodules irrespective of shape, size and distribution of gray levels. The template parameters are estimated automatically from the segmented data (after the first two steps of our CAD system for automatic nodule detection) - no a priori learning of the parameters density function is needed. We show quantitatively that this template modeling approach drastically reduces the number of false positives in the nodule detection (the third step of our CAD system for automatic nodule detection), thus improving the overall accuracy of CAD systems. We compare the performance of this approach with other approaches in the literature and with respect to human experts. The impact of the new template model includes: 1) flexibility with respect to nodule topology - thus various nodules can be detected simultaneously by the same technique; 2) automatic parameter estimation of the nodule models using the gray level information of the segmented data; and 3) the ability to provide exhaustive search for all the possible nodules in the scan without excessive processing time - this provides an enhanced accuracy of the CAD system without increase in the overall diagnosis time.
\end{abstract}

\section{Introduction}

Lung cancer screening is a major research activity worldwide. The goal is to design a CAD system that will contribute to early diagnosis of lung cancer, a major cause of death in various parts of the world. The work of our group uses helical low dose thin slice $(2 \mathrm{~mm}-2.5 \mathrm{~mm})$ chest CT scanning (LDCT) 
which provides very high spatial, temporal, and contrast resolution of anatomic structures. Automated detection of lung nodules in thoracic CT scans is an important clinical challenge, especially because manual analysis by a radiologist is time-consuming and may result in missed nodules. Furthermore, the amount of image data to be analyzed continues to increase. We will not attempt an exhaustive search on this subject because of the enormous size of literature on early diagnosis of lung cancer, thus we will limit our scope to the image analysis aspect of the CAD research on this subject. Specifically, we will focus on the automatic detection and identification of lung nodules from the LDCT scanning. Only sample studies will be referred to below as they pertain to our work.

Most of the CAD work in lung cancer screening involves two-stage detection of lung nodules, such that initial candidate nodules are first selected and then the false ones, called false positive nodules (FPNs), are partially eliminated while preserving the true positive nodules (TPNs). For example, the conformal nodule filtering [1] or unsharp masking [2] enhance nodules and suppress other structures at the first stage in order to separate the candidates from the background by simple thresholding. To improve the separation, the background trend is corrected within image regions of interest [3. A series of $3 \mathrm{D}$ cylindrical and spherical filters are used to detect small lung nodules from high resolution CT images [4]. Circular nodule candidates are detected by template matching [2, 5, 6] or other pattern recognition techniques such as fuzzy clustering [7, linear discriminant functions [8], rule-based classification [9], and patient-specific a priori model [10]. Also, cylindrical vascular models are used along with spherical nodular ones to amplify the template matching [1].

The FPNs are excluded at the second stage by feature extraction and classification [12, 13]. Such features as circularity, size, contrast [12, or local curvature [13] are extracted by morphological techniques, and artificial neural networks (ANN) are frequently used as post-classifiers [14.

Our CAD system detects the nodules in LDCT images in three main steps: 1) segmentation of the raw scanning information to isolate the lung tissues from the rest of the structures in the chest cavity [Figs. 11(a) and (b)];2) extraction of the 3D anatomic structures (e.g., blood vessels, bronchioles, alveoli, etc., and possible abnormalities) from the already segmented lung tissues [Fig. 11(c)]; and 3) identification of the nodules by isolating the true nodules from other extracted

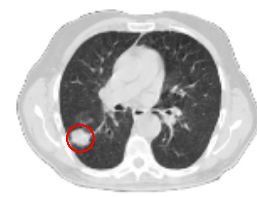

(a)

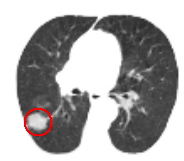

(b)

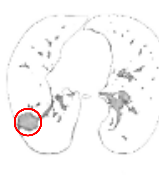

(c)

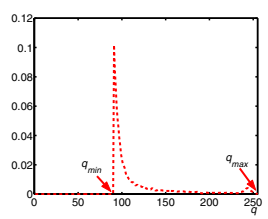

(d)

Fig. 1. First two segmentation steps: (a) an initial LDCT slice, (b) separated lung regions, (c) extracted objects (a nodule candidate is encircled), and (d) an empirical marginal gray level distribution for the objects (here, the gray range is $[98,255]$ ) 
structures. The first two steps considerably reduce the search space by using segmentation algorithms based on representing each CT slice as a sample of a Markov-Gibbs random field of region labels and gray levels. Details of the algorithms are presented in [15].

In this paper we focus on the third step that detects and classifies the nodules among the extracted 3D structures. Both the nodules and normal objects have almost the same marginal gray level distributions similar to that in Fig. 11(d) for all the extracted objects in Fig. 11(c). Therefore, segmentation based solely on gray level distribution (e.g, thresholding) will not work; we need to include geometrical/shape information in this process. The approach proposed in this paper includes a $3 \mathrm{D}$ deformable nodule prototype combined with a central-symmetric $3 \mathrm{D}$ intensity model of the nodules. The model closely approximates an empirical marginal probability distribution of image intensities in the real nodules of different size and is analytically identified from the empirical distribution.

\section{Detecting Lung Nodules with Deformable Prototypes}

The detection step extracts, by shape and intensities, and classifies the nodule candidates among all the 3D objects selected at the second segmentation stage.

\subsection{Deformable Prototype of a Candidate Nodule}

To extract the nodule candidates among the already selected objects like those in Fig. 11(c), we use the deformable prototypes generated by level sets [16] that have become a powerful segmentation tool in recent years. The evolving prototype's surface at time instant $t^{\circ}$ is a propagating zero-level front $\phi\left(x, y, z, t^{\circ}\right)=0$ of a certain $4 \mathrm{D}$ scalar function $\phi(x, y, z, t)$ of $3 \mathrm{D}$ Cartesian co-ordinates $(x, y, z)$ and time $t$. Changes of $\phi$ in continuous time are given by the partial differential equation:

$$
\frac{\partial \phi(x, y, z, t)}{\partial t}+F(x, y, z)|\nabla \phi(x, y, z, t)|=0
$$

where $F(x, y, z)$ is a velocity function and $\nabla=\left[\frac{\partial}{\partial x}, \frac{\partial}{\partial y}, \frac{\partial}{\partial z}\right]^{\top}$. The scalar velocity function controlling the front evolution depends on local geometric properties, e.g. a local curvature, $k(x, y, z)$, of the front, and on local input data parameters, e.g. a 3D gradient, $\nabla I(x, y, z)$, of the segmented 3D image $I$.

In practice, the difference relationship replaces Eq. (1) and each next value $\phi\left(x, y, z, t_{n+1}\right)$ relates to the current one $\phi\left(x, y, z, t_{n}\right)$ at respective time instants $t_{n+1}$ and $t_{n}$ such that $t_{n+1}-t_{n}=\triangle t ; n=0,1, \ldots$, as follows: $\phi\left(x, y, z, t_{n+1}\right)=$ $\phi\left(x, y, z, t_{n}\right)-\triangle t \cdot F(x, y, z)\left|\nabla \phi\left(x, y, z, t_{n}\right)\right|$. The velocity function $F$ plays a major role in the propagation process. Among variants in [17, 18, we have chosen $F(x, y, z)=-h(x, y, z)(1+\varepsilon k(x, y, z))$ where $h(x, y, z)$ and $\varepsilon$ are a local consistency term and a smoothing factor, respectively. Since the level set for a segmented 3D image $I$ can always be initialized inside an object, an appropriate consistency term to evolve faster to the object boundary can be as follows: 


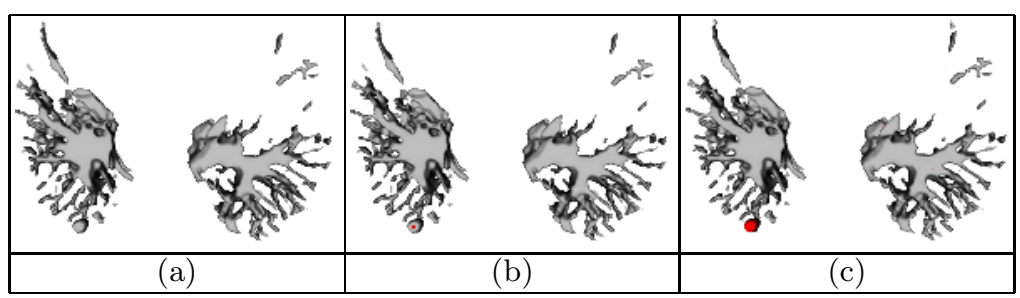

Fig. 2. Part of the separated 3D lung objects (a), the initialized level set (b) indicated by red color, and the finally extracted potential nodule candidate (c)

$h(x, y, z)=(1+|\nabla I(x, y, z)|)^{-1}$. To keep the level set front from propagating through blood vessels to which the nodules may be connected, we apply a lowpass filter after each propagation step $n$. Figure 2 shows results of extracting a potential nodule candidate with the deformable prototype. To check whether the extracted object is really a nodule candidate, we should measure similarity between grayscale patterns in the extracted part of the initial 3D image and the intensity prototype of the nodule of that shape.

\subsection{Similarity Measure for Grayscale Nodule Prototypes}

Analysis of abnormalities in real 3D LDCT slices suggests that gray levels in central cross-sections of a solid-shape 3D nodule or in a solid-shape 2D nodule roughly follow a central-symmetric Gaussian spatial pattern such that the large central intensity gradually decreases towards the boundary. Moreover, the marginal gray level distributions for all 3D objects separated from the lung tissues at the second segmentation stage (e.g. arteries, veins, bronchi, or nodules of different size) are very similar to each other. The 3D Gaussian intensity pattern in each grayscale nodule prototype ensures that the marginal gray level distribution closely approximates the empirical one for each real nodule in the LDCT data.

Let the prototype be a central-symmetric 3D Gaussian of radius $R$ with the maximum intensity $q_{\max }$ in the center so that the gray level $q(r)$ at any location $(x, y, z)$ at radius $r=\left(x^{2}+y^{2}+z^{2}\right)^{\frac{1}{2}}$ with respect to the center $(0,0,0)$ is given by the obvious relationship:

$$
q(r)=q_{\max } \exp \left(-(r / \rho)^{2}\right) ; \quad 0 \leq r \leq R
$$

The scatter parameter $\rho$ in Eq. (2) specifies how fast the signals decrease towards the boundary of the prototype. The maximum gray level, $q_{\max }=q(0)$, and the minimum one, $q_{\min }=q(R)$, on the boundary of the spherical Gaussian prototype of the radius $R$ uniquely determine this parameter as follows:

$$
\rho=R\left(\ln q_{\max }-\ln q_{\min }\right)^{-\frac{1}{2}}
$$


Because all the prototype's points with a fixed gray value $q$ in the continuous interval $\left[q_{\min }, q_{\max }\right]$ are located at the spherical surface of the radius $r(q)=$ $\rho\left(\ln q_{\max }-\ln q\right)^{\frac{1}{2}}$, their density is proportional to the surface area $4 \pi r^{2}(q)$. Therefore, the marginal probability density function for such a prototype is $\psi(q)=\gamma r^{2}(q)$ where $\gamma$ is the normalizing factor such that $\int_{q_{\min }}^{q_{\max }} \psi(q) d q=1$. It is easily shown that this function has the following closed form:

$$
\psi\left(q \mid q_{\min }, q_{\max }\right)=\frac{\ln q_{\max }-\ln q}{q_{\max }-q_{\min }\left(1+\ln q_{\max }-\ln q_{\min }\right)}
$$

The gray level parameters $q_{\max }$ and $q_{\min }$ are estimated from the empirical marginal distribution for each segmented $3 \mathrm{D}$ object, e.g. $q_{\max }=255$ and $q_{\min }=$ 98 for the objects extracted in Fig. 1,

To evaluate similarity, the gray level nodule prototype is centered at the centroid of the volume extracted with the deformable prototype.

\subsection{Lung Nodule Detection Algorithm}

1. Separate lung regions from a given CT scan using the segmentation algorithms in [15]; see an example in Fig. 11(b).

2. Separate arteries, veins, bronchi, bronchioles, and lung nodules (if they exist) from the above lung regions using the same segmentation algorithms in [15]; see an example in Fig. 11(c).

3. From the empirical marginal gray level distribution for the objects separated at Step 2 calculate $q_{\min }$ and $q_{\max }$; see an example in Fig. 1(d).

4. Stack all the voxels separated at Step 2

5. Pop-up a top voxel from the stack as a seed for the deformable prototype and let this latter propagate until reaching a steady state indicating that the voxel set $\mathbf{U}$ enclosed by the final prototype constitutes an extracted object.

6. Calculate the centroid for the voxel set $\mathbf{U}$ extracted at the previous step; find the maximum, $R_{\max }$, and the minimum, $R_{\min }$, radii from the centroid of the boundary of that set; find the average radius $R=\left(R_{\min }+R_{\max }\right) / 2$, and estimate the scatter parameter $\rho$ from Eq. (3).

7. Use Eq. (2) to assign the prototype gray levels $N_{x, y, z}$ for each extracted $\operatorname{voxel}(x, y, z) \in \mathbf{U}$.

8. Use the normalized cross-correlation $\operatorname{Corr}_{\mathbf{C}, \mathbf{N}}$ between the actual extracted object $\mathbf{C}=\left[C_{x, y, z}:(x, y, z) \in \mathbf{U}\right]$ and its gray level nodule prototype $\mathbf{N}=\left[N_{x, y, z}:(x, y, z) \in \mathbf{U}\right]$ as the similarity measure (see Fig. 33).

9. If Corr $\operatorname{Cos}_{\mathbf{N}} \geq \tau$ where $\tau$ is a pre-selected similarity threshold (in our experiments below we set it to $\tau=0.85$ on the empirical basis), then classify the extracted object as the potential nodule candidate.

10. Remove all the voxels of the extracted object from the stack.

11. If the stack is empty then stop, otherwise go to Step 5]

To reduce the error rate, the initially selected potential candidates are postclassified just as in [19] to distinguish between the false (FPNs) and true (TPNs) 

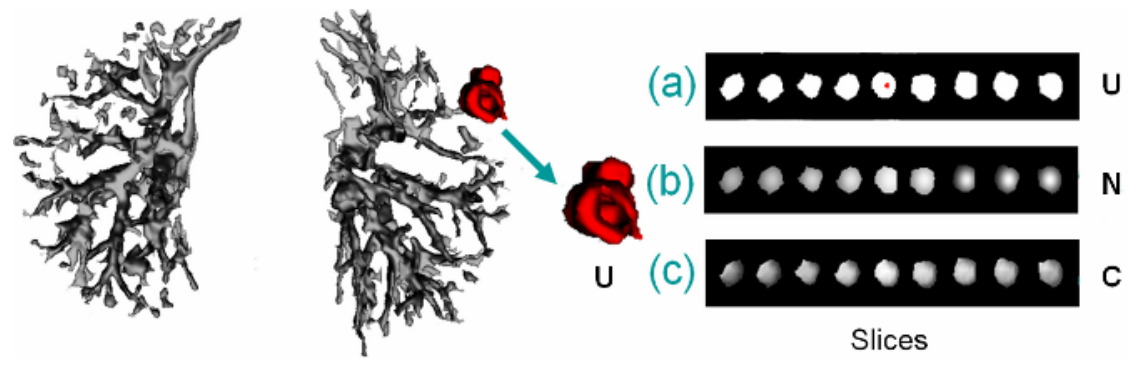

Slices

Fig. 3. Detected nodule candidate: (a) slices of the extracted voxel set $\mathbf{U}$, (b) the gray level prototype $\mathbf{N}$, and (c) the actual gray levels $\mathbf{C}$; the correlation $\operatorname{Corr}_{\mathbf{C}, \mathbf{N}}=0.886$

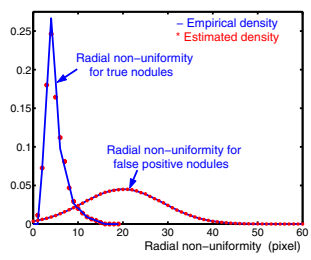

(a)

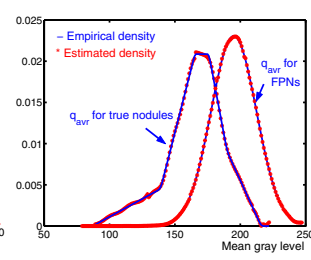

(b)

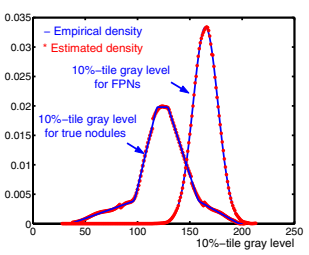

(c)

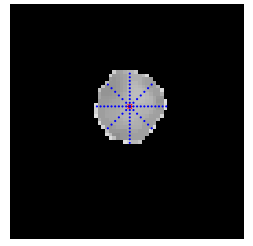

(d)

Fig. 4. Estimated and empirical distributions for radial non-uniformity (a), mean gray level (b), and 10\%-tile gray level(c), and the radii $d_{\theta}$ in 8 directions $\theta$ from the centroid to the boundary that specify the radial non-uniformity $\max _{\theta}\left(d_{\theta}\right)-\min _{\theta}\left(d_{\theta}\right)$

positive nodules. In contrast to [19], probability distributions of each feature required by the classifier are accurately estimated with linear combinations of Gaussians (LCGs) using our algorithms in [15]. The LCGs approximate the empirical distributions for a training set of the nodules. Figure 4 shows the empirical and estimated distributions of each feature for both TPNs and FPNs.

\section{Experimental Results and Conclusions}

The proposed algorithm was tested on the same LDCT scans of 50 screened subjects. Among them, 16 subjects had abnormalities in their CT scans and 34 subjects were normal (this classification was validated by two radiologists). The chest CT data used in this paper were obtained from Mansoura University, Urology and Nephrology Center, Radiology department, Mansoura, Egypt as follows: 1) The scanner: Multidetecor scanner (Light speed plus; GE), 2) Scan mode: Helical, 3) Slice thickness: 2.5mm, 4) Field of view: large, 5) K.V.: 120, 6) M.A.: 200, 7) Exposure time: 20-25 sec., and 8) Window level: -500 \& length 1500. Our approach extracted 113 potential nodule candidates out of the true 119 nodules and 14 FPNs. The post-classification has reduced the number of FPNs to 4 but simultaneously rejected two true nodules. Thus, the final detection rate of the TPNs was $93.3 \%$ (111 out of 119 ) with the FPNs rate of $3.36 \%$. Both the 


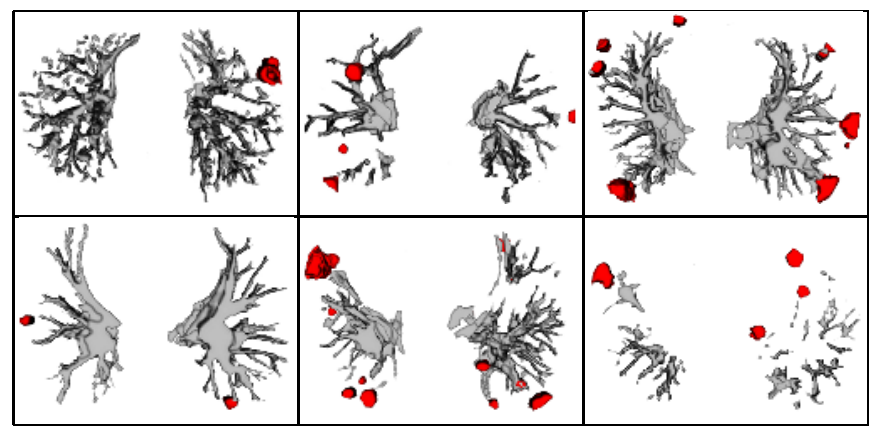

Fig. 5. Large candidate nodules (shown in the red colour) detected with our approach

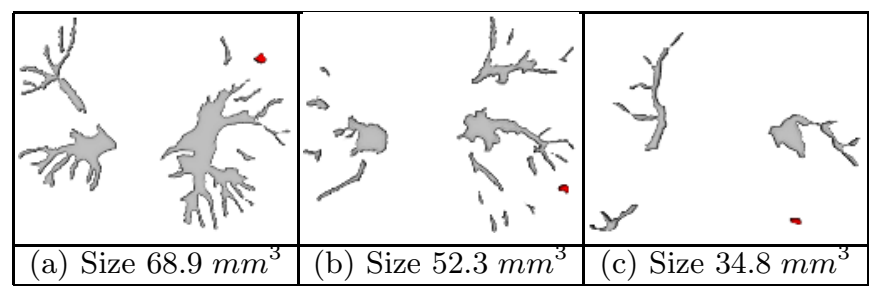

Fig. 6. Small candidate nodules (shown in the red colour) detected with our approach

Table 1. Recognition of different abnormalities with our present algorithm, the previous one in 19, and the algorithm in [5] w.r.t. the "ground truth" by two radiologists (the corresponding numbers are bold, bold italic, italic, and regular, respectively)

\begin{tabular}{|c|c|c|c|c|}
\hline $\begin{array}{c}\text { Type of the } \\
\text { nodules }\end{array}$ & $\begin{array}{c}\text { True detected } \\
\text { nodules before } \\
\text { removing FPNs }\end{array}$ & $\begin{array}{c}\text { False detected } \\
\text { nodules before } \\
\text { removing FPNs }\end{array}$ & $\begin{array}{c}\text { True detected } \\
\text { nodules after } \\
\text { removing FPNs }\end{array}$ & $\begin{array}{c}\text { False detected } \\
\text { nodules after } \\
\text { removing FPNs }\end{array}$ \\
\hline Lung wall & $\mathbf{2 8 : 2 8 : 1 4 : 2 9}$ & $\mathbf{3}: \mathbf{8}: 86$ & $\mathbf{2 8}: 27: 13: 29$ & $\mathbf{1}: 2: 17$ \\
Calcified & $\mathbf{4 9}: \mathbf{4 6}: 31: 49$ & $\mathbf{2}: \mathbf{4}: 35$ & $\mathbf{4 8}: \mathbf{4 6}: 31: 49$ & $\mathbf{0}: \mathbf{1}: 9$ \\
Non-calcified & $\mathbf{1 7}: \mathbf{1 2}: 14: 18$ & $\mathbf{3}: \mathbf{5}: 25$ & $\mathbf{1 7}: \mathbf{1 2}: 14: 18$ & $\mathbf{1}: \mathbf{3}: 14$ \\
Small & $\mathbf{1 9}: \mathbf{1 7}: 10: 23$ & $\mathbf{6}: \mathbf{2 5}: 34$ & $\mathbf{1 8}: \mathbf{1 5}: 9: 23$ & $\mathbf{2}: \mathbf{5}: 12$ \\
\hline \multicolumn{4}{c}{ Total TPN rate } & $\mathbf{9 3 . 3 \%}: \mathbf{8 2 . 3 \%}: 56.3 \%$ \\
Total FPN rate & $\mathbf{3 . 4 \% : 9 . 2 \% : 4 3 . 6 \%}$ \\
\hline
\end{tabular}

rates are notably better than $82.3 \%$ and $9.2 \%$ in [15], respectively. Figure 5] shows several large lung nodules detected by our approach, and a few small detected TPNs are depicted in Fig. 6.

Table 1 presents the above results in detail and also compares them to the algorithm in [5], the only work known to us on detecting the lung nodules from the LDCT scans using template matching. It uses fixed circular, semicircular and approximate spherical templates with parameters which are manually derived from a training data set. This algorithm, after the post-classification, gives the 
overall TPN rate of $56.3 \%$ (67 out of 119 ) with the FPN rate of $43.6 \%$. It is felt that this algorithm fails to detect a large number of the true nodules because of the fixed-size templates and manual specification of their gray level patterns. At times these patterns change from one LDCT slice to another depending on the scanned cross section and internal organs that appear in that cross section. In our present algorithm, these patterns are analytically adjusted to each extracted shape by applying Eq. (3) to each CT slice.

These experiments show that our new deformable level-set prototype with the analytically modeled standard intensity pattern detects more than $90 \%$ of the true lung abnormalities. The overall processing time for the data set of size $1005 \times 804 \times 186$ is 6,18 , and $16 \mathrm{~min}$. for the proposed approach and the algorithms in [19, 5], respectively. But it is still difficult to accurately detect very small lung nodules similar to bronchi and bronchioles. Our future work is focused on features that distinguish between the small lung nodules and normal objects. Also, we are going to analyze the accuracy of our CAD system with respect to the ground truth on a much larger number of the real LDCT scans.

\section{References}

1. S. C. B., M. T. Freedman, J. S. Lin, and S. K. Mun, "Automatic lung nodule detection using profile matching and back-propagation neural network techniques," J. Digital Imaging, Vol. 6, pp. 48-54, 1993.

2. F. Mao, W. Qian, J. Gaviria, and L. Clarke, "Fragmentary window filtering for multiscale lung nodule detection, Academic Radiology, Vol. 5, pp. 306-311, 1998.

3. X. Xu, S. Katsuragawa, K. Ashizawa,H. MacMahon, and K. Doi, "Analysis of image features of histograms of edge gradient for false positive reduction in lung nodule detection in chest radiographs," Proc. SPIE, Vol. 3338, pp. 318-326, 1998.

4. S. Chang, H. Emoto, D. N. Metaxas, and L. Axe, "Pulmonary Micronodule detection from 3D Chest CT," Proc. MICCAI, Saint-Malo, France, Sept. 26-29, 2004, pp. 821-828, 2004.

5. Y. Lee, T. Hara, H. Fujita, S. Itoh, and T. Ishigaki, Automated Detection of Pulmonary Nodules in Helical CT Images Based on an Improved Template-Matching Technique, IEEE Trans. on Medical Imaging, Vol. 20, pp. 595-604, 2001.

6. A. El-Baz, A. A. Farag, R. Falk, and R. La Rocca, "Automatic Identification of Lung Abnormalities in Chest Spiral CT Scans," International Conference on Acoustics, Speech, and Signal Processing, ICASSP-03, April 6-10, 2003, pp. 261264.

7. K. Kanazawa, Y. Kawata, N. Niki, H. Satoh, H. Ohmatsu, R. Kakinuma, M. Kaneko, N. Moriyma, and K. Eguchi, "Computer-aided diagnosis for pulmonary nodules based on helical CT images," Computerized Medical Imaging and Graphics, vol. 22, pp. 157-167, 1998.

8. Y. Kawata, N. Niki, H. Ohmatsu, M. Kusumoto, R. Kakinuma, K. Mori, H. Nishiyama, K. Eguchi, M. Kaneko, N. Moriyama, "Computer-Aided Diagnosis of Pulmonary Nodules Using Three-Dimensional Thoracic CT Images," Proc. MICCAI, Utrecht, Netherlands, October 14-17, 2001, pp. 13931394, 2001.

9. R. M. Betke and J. P. Ko, "Detection of Pulmonary Nodules on CT and Volumetric Assessment of Change over Time," Proc. MICCAI, Cambridge, UK, 1999, pp. 245252, 1999. 
10. M. S. Brown, M. F. McNitt-Gray, J. G. Goldin, R. D. Suh, J. W. Sayre, and D. R. Aberle "Patient-Specific Models for Lung Nodule Detection and Surveillance in CT Images," IEEE Trans. on Medical Imaging, vol. 20, pp. 1242-1250, 2001.

11. H. Takizawa, K. Shigemoto, S. Yamamoto, T. Matsumoto, Y. Tateno, T. Iinuma and M. Matsumoto, "A Recognition Method of Lung Nodule Shadows in X-Ray CT Images Using 3D Object Models," Int. J. of Image and Graphics, vol. 3, pp. 533$545,2003$.

12. X. Xu et al., S. Katsuragawa, K. Ashizawa,H. MacMahon, and K. Doi, "Analysis of image features of histograms of edge gradient for false positive reduction in lung nodule detection in chest radiographs, Proc. SPIE, Vol. 3338, pp. 318-326, 1998.

13. M. J. Carreira, D. Cabello, M. G. Penedo,and J. M. Pardo, "Computer aided lung nodule detection in chest radiography," Proc. Conf. Image Analysis Applications and Computer Graphics, Berlin, pp. 331-338, 1995.

14. S. C. B. Lo, S. L. A. Lou, J. S. Lin, M. T. Freedman, M. V. Chien, and S. K. Mun, "Artificial convolution neural network techniques and applications for lung nodule detection," IEEE Trans. on Medical Imaging, vol. 14, 1995, pp. 711-718.

15. G. Gimel'farb, A. A. Farag, and A. El-Baz, "Expectation-Maximization for a linear combination of Gaussians", in Proc. IAPR Int. Conf. Pattern Recognition (ICPR 2004), Cambridge, UK, August 23-26, 2004, vol.2, pp. 422-425, 2004.

16. J.A. Sethian, Level Set Methods and Fast Marching Methods, Cambridge University Press, USA, 1999.

17. J. Gomes and O. Faugeras, Reconciling distance functions and level-sets. Technical Report 3666, INRIA, April 1999.

18. N. Paragios and R. Deriche, "Unifying boundary and region-based information for geodesic active tracking," Proc. CVPR, Fort Collins, Colorado, June 1999, Vol. 2, pp. 300-305, 1999.

19. A. A. Farag, A. El-Baz, G. Gimel'farb, R. Falk, and S. G. Hushek, "Automatic detection and recognition of lung abnormalities in helical CT images using deformable templates," Proc. MICCAI, Saint-Malo, France, Sept. 26-29, 2004, pp. 856-864, 2004. 